\title{
TECHIJSAL DOUES
}

\section{Selecting Letter Sizes for Technical Data Slides}

\section{B. J. WALKOWIAK AND R. E. RIES}

Highlight: Careful selection of letter size and amount of information to be included before photographing slides is essential for legible slides. This article presents information that can be used to select the correct size of lettering for a specific drawing size before drafting and photographing them so as to insure that the $2 \times 2$ inch slides produced will be legible even to those sitting in the back of the audience.

Authors are surface compliance specialist, Bureau of Land Management, Casper, Wyoming 82601 and range scientist, Northern Great Plains Research Center, Agricultural Research Service, U.S. Department of Agricul ture, Mandan, North Dakota 58554
Most photographs or drawings that are prepared for manuscripts do not make good slide copying material (Smith 1957; Woolfolk 1963). Manuscript copy reduced to slide size is too small to be legible and will cause viewers to lose interest. To increase audience appeal and interest, a good slide must stress a single point clearly and quickly. Therefore, slide copying material must be planned carefully before photographing.

In general, if you can readily and easily read the lettering on a slide held at normal reading distance, it will be legible under most conditions to all members of an audience. Even though there is some variability between individuals in how this nule of thumb is interpreted and perceived, it has proven to be very useful (Beeler
1974). The information contained in Figure 1 provides the opportunity to apply this traditional rule and to determine letter sizes objectively before drafting and photographing the material to insure proper letter sizes on the finished slide.

\section{Methods and Discussion}

Ten different sizes of progressively larger letter sizes were used in Figure 1 with eight different sized margins. By moving the camera farther away from each successive sized drawing, we duplicated what happens to each slide as the drawing is amplified from $4 \times 6$ inches $(10.2 \times 15.2$ $\mathrm{cm})$ to $16 \times 24$ inches $(40.6 \times 61.0 \mathrm{~cm})$. The first frame of Figure 1 shows a $3 \times$ 4.5 inches $(7.6 \times 11.4 \mathrm{~cm})$ photograph of a typed card reduced to slide size. The
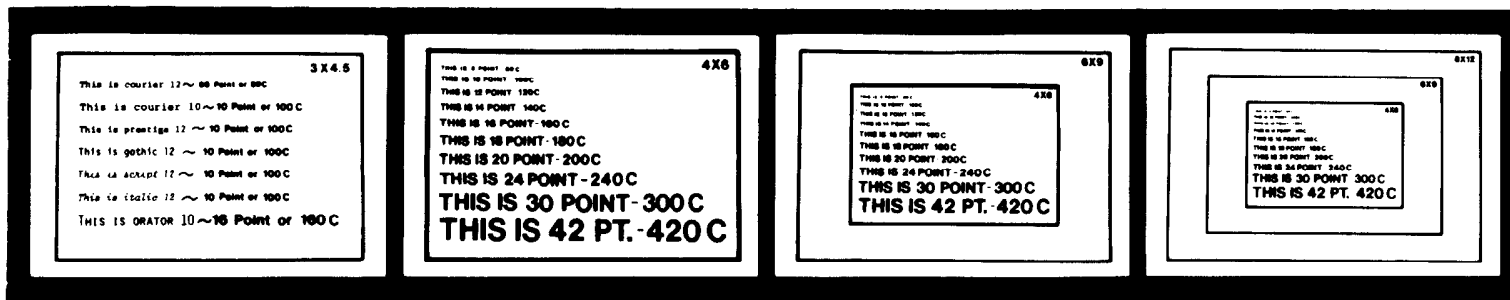

$3 \times 4.5 \mathrm{in}$.

$4 \times 6$ in.

$6 \times 9$ in.

$8 \times 12$ in.
$7.6 \times 11.4 \mathrm{~cm}$.
$10.2 \times 15.2 \mathrm{~cm}$.
$15.2 \times 22.9 \mathrm{~cm}$.
$20.3 \times 30.5 \mathrm{~cm}$.
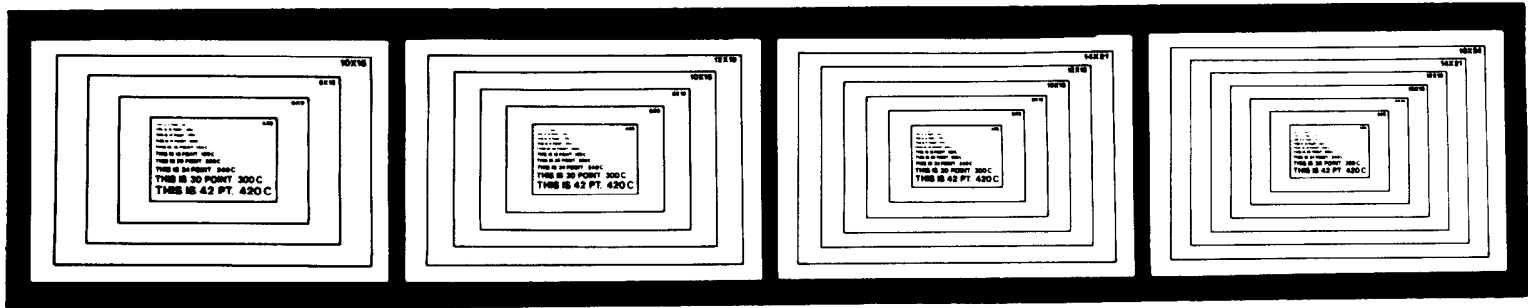

$10 \times 15 \mathrm{in}$. $25.4 \times 38.1 \mathrm{~cm}$.
$12 \times 18$ in. $30.5 \times 45.7 \mathrm{~cm}$.
$14 \times 21$ in. $35.6 \times 53.3 \mathrm{~cm}$.
$16 \times 24$ in. $40.6 \times 61.0 \mathrm{~cm}$.

Fig. 1. Actual slide size. The lettering style is helvetica medium for both the transfer lettering and letter template. 


\section{MINIMUM LETTER SIZE}

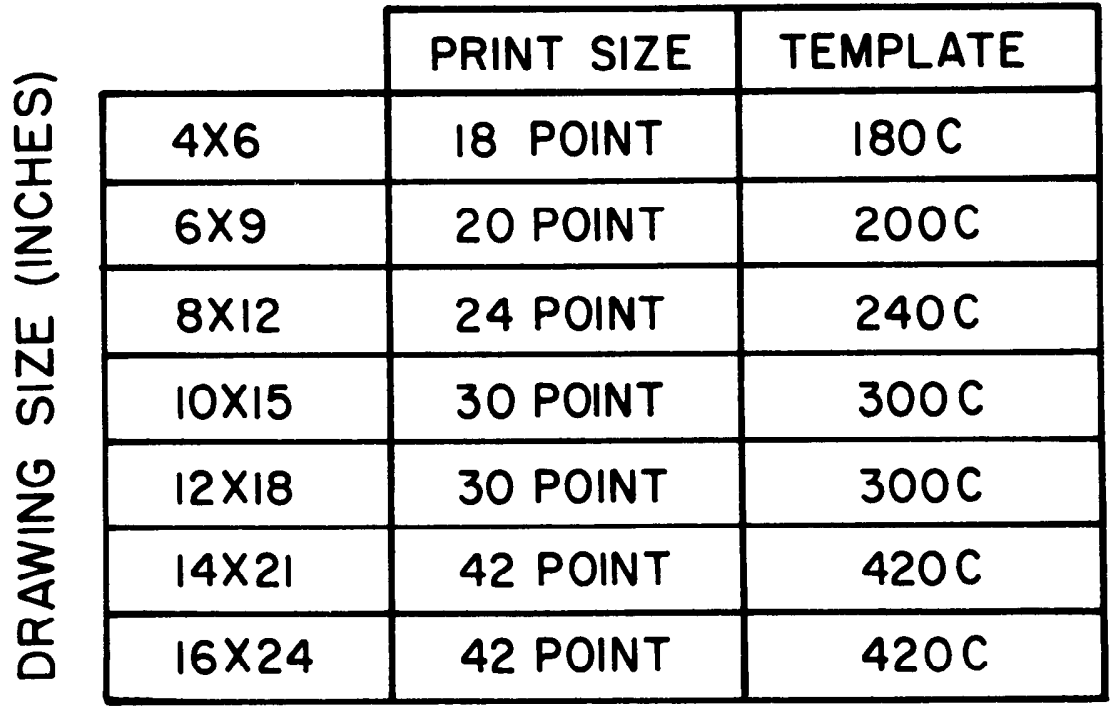

Fig. 2. Recommended minimum letter sizes for each drawing size.

maximum number of typing spaces for each line is $\mathbf{5 4}$ for elite and $\mathbf{4 5}$ for pica (Eastman Kodak 1975). The maximum information space is $3 \times 4.5$ inches $(7.6 \times 11.4 \mathrm{~cm})$ for a $35-\mathrm{mm}$ camera, which has a height to width ratio of 2:3 respectively. In Figure 1 for example, it is possible to read all the lines in the $4 \times 6$ inches $(10.2 \times 15.2 \mathrm{~cm})$ frame. However, by allowing your eyes to scan the whole slide, 18 point lettering
(Leroy size $180 \mathrm{C}$ ) is the first size you can comfortably see. Therefore, for slide preparation from a $4 \times 6$ inch $(10.2 \times 15.2 \mathrm{~cm})$ drawing, 18 point lettering should be the smallest sized lettering used. As the drawing size is increased to $6 \times 9$ inches $(15.2 \times 22.9 \mathrm{~cm})$, the 18 point lettering is no longer easily read and 20 point lettering becomes the new minimum letter size. As the drawing size is increased, so must the letter size (Eastman Kodak 1975). When using Figure 1, measure the actual picture to be photographed and not the paper size. Proper selection of lettering shown in Figure 1 will assist a draftsman in selecting an optimum letter size.

The most favorable and versatile letter sizes, as viewed by the authors, are summarized in Figure 2. Under some conditions, you may wish to deviate from the lettering shown in Figure 2 and reduce or enlarge the letter sizes. However, a change in letter sizes can adversely affect the versatility or the legibility of the slides. Examples of where reduced letter sizes may be desirable would be when (a) projection facilities are outstanding, like room size, projector location, and area of projections; (b) when deemphasizing particular data; and (c) when large quantities of information must be included. Some examples of where enlarging letter sizes might be desirable would be for (a) titling; (b) emphasizing; and (c) very poor projection facilities.

\section{Literature Cited}

Beeler, Robert. 1974. Titling techniques. Book let AE-83. Eastman Kodak Co. Standard Book Number 0-87985-044-2.

Eastman Kodak. 1975. Planning and producing slide programs. Booklet S-30. Standard Book Number 0-87985-150-3.

Smith, Henry W. 1957. Presenting information with 2 by 2 slides. Agron. J. 49:108-113.

Woolfolk, E. J. 1963. Editorial. The last and next annual meeting. J. Range Manage. 16: 103-105.

\section{Impact of Herbivores}

\section{on Arid and Semiarid Rangeland Ecosystems-} Proceedings of the Second Workshop of the US/AUSTRALIA RANGELANDS PANEL-Adelaide, 1972.

We have received a small number of copies of the above Proceedings on consignment from the Australian Rangeland Society, publishers of the Second Workshop. These copies are available from the Society for Range Management, 2760 West Fifth Avenue, Denver, Colo. 80204, for approximately $\$ 7.50$ (US dollars). The publication contains the contributions of the 10 United States and 23 Australian scientists participating in the Second Workshop.

Also available from the SRM Denver headquarters is a limited supply of Arid Shrublands-Proceedings of the Third Workshop of the US/ AUSTRALIA RANGELANDS PANEL, Tucson, Ariz. 1973. (\$3.00 postpaid)

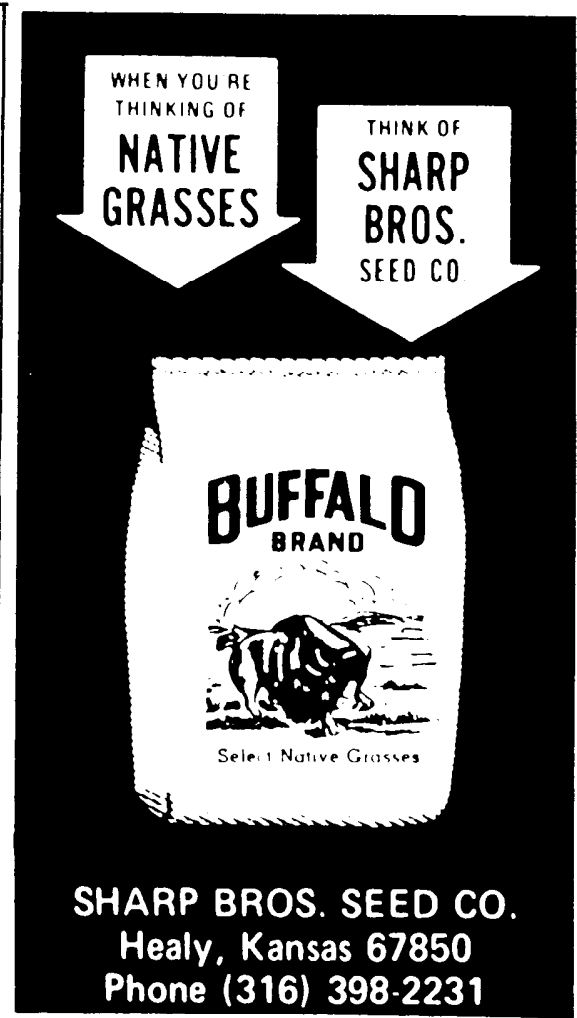

